\title{
Effect of Scattering Correction in Neutron Imaging of Hydrogenous Samples using the Black Body Approach
}

\author{
Chiara Carminati $^{1, a^{*}}$, Pierre Boillat ${ }^{1,2}$, Sarah Laemmlein ${ }^{3}$, Petra Heckova ${ }^{4}$, \\ Michal Snehota ${ }^{4}$, David Mannes ${ }^{1}$, Jan Hovind ${ }^{1}$, Markus Strobl ${ }^{1}$ \\ and Anders Kaestner ${ }^{1}$ \\ ${ }^{1}$ Laboratory for Neutron Scattering and Imaging, Paul Scherrer Institut, CH-5232 Villigen-PSI \\ ${ }^{2}$ Electrochemistry Laboratory, Paul Scherrer Institut, CH-5232 Villigen-PSI \\ ${ }^{3}$ Cellulose \& Wood Materials, Swiss Federal Laboratories for Materials Science and Technology \\ (EMPA), CH-8600 Duebendorf, Switzerland \\ ${ }^{4}$ University Centre for Energy Efficient Buildings, Czech Technical University in Prague, \\ Bustehrad, Czech Republic \\ ${ }^{a}$ chiara.carminati@psi.ch
}

Keywords: Scattering Artefacts, Systematic Biases, Quantitative Neutron Imaging

\begin{abstract}
The "black body" (BB) method is an experimental approach aiming at correcting scattering artifacts and systematic biases from neutron imaging experiments. It is based on the acquisition of reference images, obtained with an interposed grid of neutron absorbers (BB), from which the background including contaminations of scattering from the sample can be extrapolated. We evaluate in this paper the effect of the BB correction on two experimental datasets acquired with different setups at the NEUTRA and ICON beamlines at the Paul Scherrer Institut. With the two experiments we demonstrate the efficient utilization of the method for 2D as well as 3D data and in particular for kinetic studies. In the first dataset, differently varnished wood samples are studied through time resolved kinetic neutron radiography to evaluate the change in wood moisture content due to changes in relative humidity. In the second case study, engineered soil sample simulating a small experimental bioretention cell with rainfall, also known as rain garden, is imaged through on-the-fly neutron tomography.
\end{abstract}

\section{Introduction}

Together with spectral influence (beam hardening) and effects at pronounced edges, scattering from the sample and the detection system poses the biggest challenge for quantitative measurements of the linear attenuation coefficients in neutron imaging experiments with high spatial resolution.

We have recently introduced an efficient method and the corresponding data treatment for scattering correction that, compared to earlier attempts, has the advantage in neither requiring prior knowledge of the neutron spectrum nor of the sample composition [1, 2]. The method is based on the acquisition of additional reference images with an interposed grid of neutron absorbers, called "black bodies" (BB) which lend the method its name: BB correction. The main idea of the approach is that the signal measured behind a black body can be interpreted as the additive background of scattering components.

Here we present exemplary applications of the method to different imaging experiments and we discuss the effect on relevant quantifications. We consider two experiments that were performed at the Paul Scherrer Institut (PSI). In the first one, a collection of wood samples with different varnishing treatments are studied through kinetic neutron radiography in order to evaluate the changes in wood moisture content due to changes in relative humidity. In the second 
case study, an engineered soil sample simulating a small experimental bioretention cell with rainfall, also known as rain garden, is imaged. First, we briefly describe the type of images which are required for the BB correction, then we present the experimental setup for the two study cases and finally we show the effect of the applied correction.

\section{BB correction}

The BB correction is an experimental approach with the aim of mitigating artifacts due to systematic biases through scattering components [1,2]. Such artifacts consist of an increased transmission signal, often especially in the center of a bulk sample, resulting in a bias towards lower computed attenuation coefficients and "cupping type" effects in tomographic reconstructions, i.e. radially decreasing attenuation coefficients towards the center.

Additional images are required for the $\mathrm{BB}$ correction: the first one is the open beam with the $\mathrm{BB}$ grid (BB-OB), the second is with the sample and the $\mathrm{BB}$ grid in the beam (BB-S). From the $\mathrm{BB}-\mathrm{OB}$ images, the systematic biases that are due to scattering by the experimental apparatus can be estimated through extrapolation between the black bodies in the grid. From the BB-S, the scattering contributions of the sample can be evaluated. Dedicated image processing was developed to interpolate for each pixel position of the field of view the neutron flux measured at the $\mathrm{BB}$ positions, thus estimating the background and scattering images $[2,3]$.

Depending on the experiment type, BB-S images can and have to be acquired at different time steps during a full experimental run. In case of kinetic radiography studies, BB-S images can be acquired prior to and/or after the non-BB images, depending on whether the sample changes sufficiently during the measurement process that it affects the scattering background. Depending on the study, correspondingly, BB-S images may need to be interleaved with the non-BB images. For tomography, a sparse tomography with regular angular steps with interposed BB is generally recommended, with a number of projections on the order of the square root of the number of projections of the conventional tomography. In case of a highly symmetrical sample, for example cylindrical, this scheme can be furthermore relaxed, and a set of BB-S images can be acquired at the same tomographic angle before and/or after the tomographic scan.

\section{Case 1: Wood}

The aim of the first experiment is to study the development of moisture content in wooden musical instruments. Eight spruce wood samples with a varnished surface were produced, in order to reproduce violin characteristics. Sample dimensions of each block were 10x10x50 mm ${ }^{3}$. The lateral surfaces were sealed to ensure that the origin of the sorption process was limited to the top and bottom surfaces of each sample.

The aim of the specific experiment was the analysis of the time dependent moisture content (MC) distribution over the wood cross section. In a climate chamber [4], the samples were put at a controlled temperature of $20^{\circ} \mathrm{C}$, with relative humidity initially set at $35 \%$. While keeping the temperature constant, relative humidity was raised up to 95\%. After $20 \mathrm{~min}$, the RH reached the 95\% level, then the $\mathrm{RH}$ was kept constant at 95\% for $5 \mathrm{~h}$. The $\mathrm{RH}$ was reduced back to 35\% again to be kept constant for another $5 \mathrm{~h}$. During this process and within this sample environment, time resolved radiographs were obtained at the thermal neutron imaging beamline NEUTRA [5] at the neutron source SINQ at PSI using a scintillator/camera detector system with a field-of-view (FOV) of $150 \times 150 \mathrm{~mm}^{2}$. The scintillator used was a $50 \mu \mathrm{m}$ thick $\mathrm{LiF} / \mathrm{ZnS}$ based screen. The camera was an Andor Neo sCMOS, where the optics was set to a FOV of 161x136 $\mathrm{mm}$ and 2560x2160 pixel chip resulting in an effective pixel size of 63.1 $\mu \mathrm{m}$. Each image in the time series featured $15 \mathrm{~s}$ exposure time, with a time increment chosen to be $5 \mathrm{~min}$. The total experiment duration for a single set of samples was $10.5 \mathrm{~h}$ during which 130 radiographs were acquired. 
Open beam and sample images with a BB grid were obtained at the beginning of the experiment, with $\mathrm{RH}$ of $35 \%$. The sample scattering and background were extrapolated and subtracted from the radiographs through an ImageJ plugin implementing the BB correction. From the normalized images, the change in wood MC at each time step was computed as: $\Delta M C_{t}=\rho_{h} \Delta d_{h} / \rho_{w} d_{w}$, where $\rho_{h}$ and $\rho_{w}$ are the densities of water and oven dry wood, $d_{w}$ is the oven dry wood thickness and finally the difference in water thickness is expressed as $\Delta d_{h}=-\ln \left(T_{t} / T_{0}\right) / \Sigma_{h}$, with $\Sigma_{h}$ being the attenuation coefficient for water with respect to the NEUTRA spectrum, and $T_{t}$ and $T_{0}$ the transmission images in the wood regions for each time steps $t$ and for the reference time 0 (when $\mathrm{RH}=35 \%$ ), respectively.

\section{Case 2: Soil}

A soil sample was taken from a filter layer of a test bed simulating a bioretention cell, also called rain garden, which is a low-impact development construction that accumulates, infiltrates and treats storm water. The sample was composed by $50 \%$ of sand, $20 \%$ of topsoil and $30 \%$ of compost. The resulting soil mixture contained $12 \%$ mass fraction of particles smaller than $2 \mu \mathrm{m}$, 14\% mass fraction of particles sized between 2 and $50 \mu \mathrm{m}$ and $74 \%$ mass fraction of particles sized between 50 and $2000 \mu \mathrm{m}$. The particle density of the soil was $2563 \mathrm{~kg} / \mathrm{m}^{-3}$.

The sample was imaged at the cold neutron imaging beamline ICON [6] at PSI using a 100 $\mu \mathrm{m}$ thick LiF/ZnS based scintillator coupled with an Andor Neo sCMOS camera with a FOV of $40 \times 40 \mathrm{~mm}^{2}$ and effective pixel size of $68 \mu \mathrm{m}$. During imaging, rainfall episodes were simulated by constant flux using heavy water as flowing fluid. Fluid drainage happened by gravity, while the inflow and outflow of fluid were balance monitored. As the drying and wetting are fast processes, the imaging technique has to be fast enough to capture the water flow. On-the-fly tomographies [7] were acquired with a continuous turning of $360^{\circ} / \mathrm{min}$. Four $360^{\circ}$ turns with 300 projections for each complete turn were performed with individual projection exposure times of $0.2 \mathrm{~s}$ (rate: $5 \mathrm{fps}$ ) and a corresponding neutron dose per projection of about 100 neutrons/pixel. $\mathrm{BB}$ images were also acquired on-the-fly at the beginning and at the end of the experiment, thus corresponding to the dry and wet conditions. CT reconstruction featuring BB correction was done in our in-house open source software MuhRec [2,3].

\section{Results}

\section{Case 1: Wood}

Fig. 1 shows the results of image data processing for the wood samples with and without BB correction in the two cases of minimal and maximal RH, i.e. 35\% and 95\%, respectively, obtained at time 0 and after 5 h. In both cases, the effect of correction is clearly visible, with lower sample transmission values for the images normalized with BB correction. The extracted change in water mass within the wood samples at each time step is shown in Figure 2. Without BB correction, the mass content is underestimated up to $30 \%$ at the point of higher MC (95\%, after $5 \mathrm{~h}$ ). These results are confirmed by the measured variation of water mass before and after the experiment with a precision balance (circle in the picture), representing the reference measure. 

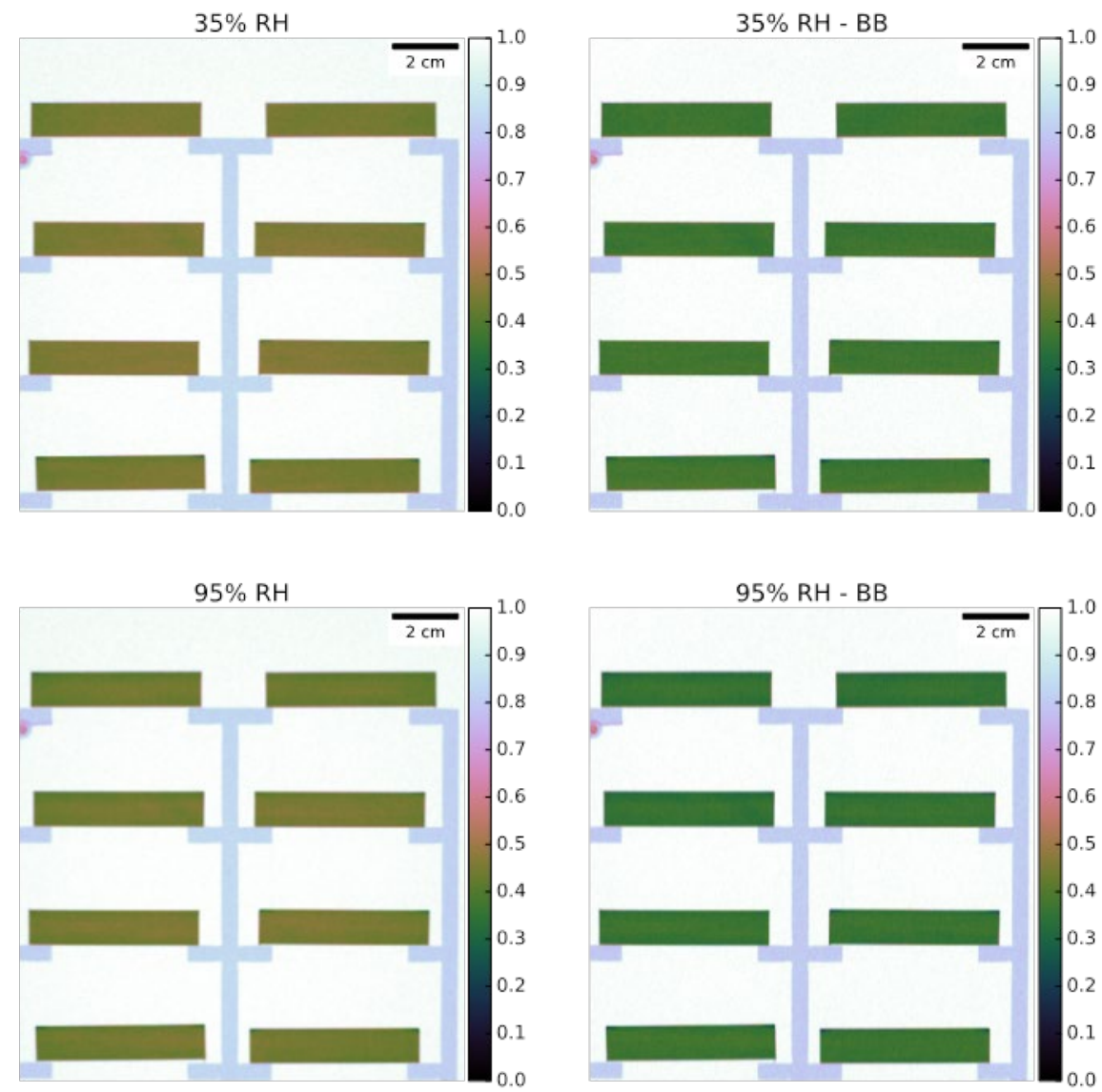

Figure 1: Transmission images computed at 35\% RH (top) and 95\% RH (bottom) without (left) and with (right) $B B$ correction

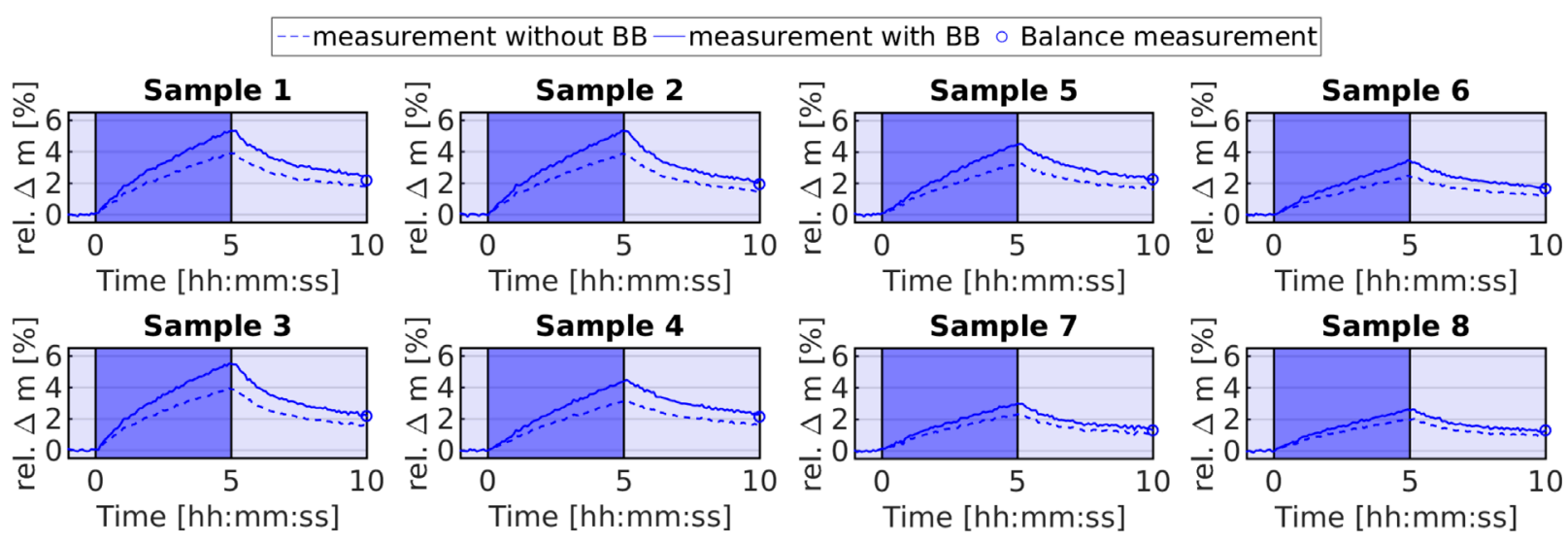

Figure 2: Relative change of water content computed from transmission images without (dashed line) and with (continuous line) BB correction, the reference balance measurement is also shown (circle). Samples 1 to 4 correspond to the top four samples, samples 5 to 8 are the four bottom samples in Fig. 1

\section{Case 2: Soil}

As a representative example of the results a cross-sectional slice at mid height of a sample reconstruction of the rain garden sample is shown in Fig.3. Even though these datasets are 
affected by a high noise level due to very low neutron statistics, attenuation coefficients are noticeably different when adopting the BB correction, for both dry and wet conditions. In particular contrast appears improved notably.

Radial mean values of attenuation coefficients plotted in Fig. 4 show that correction with the BB approach results in higher attenuation coefficient (between 5 and 14\%) for all radial position. A cupping effect does not appear prominent in the results, due to the small sample size and the in-homogeneity of the sample. However, when plotting the percent difference between the radial mean values obtained without and with BB correction (fig 4, right panels) with respect to the radial distance from the sample center, a pronounced decrease in difference when moving away from the sample center, as typical of a cupping effect, can be observed and as expected more pronounced for the wet condition.
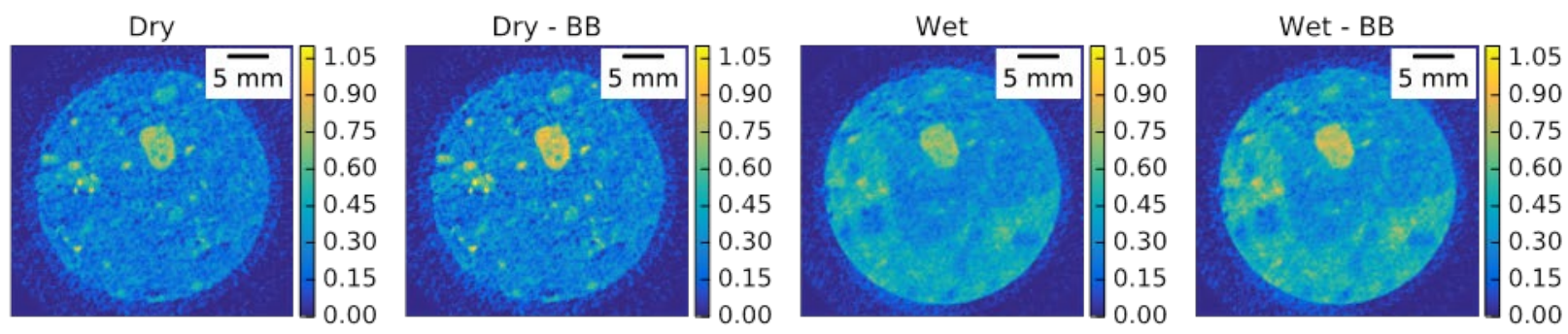

Figure 3: Reconstructed CT of the rain garden sample at dry and wet conditions without and with BB correction
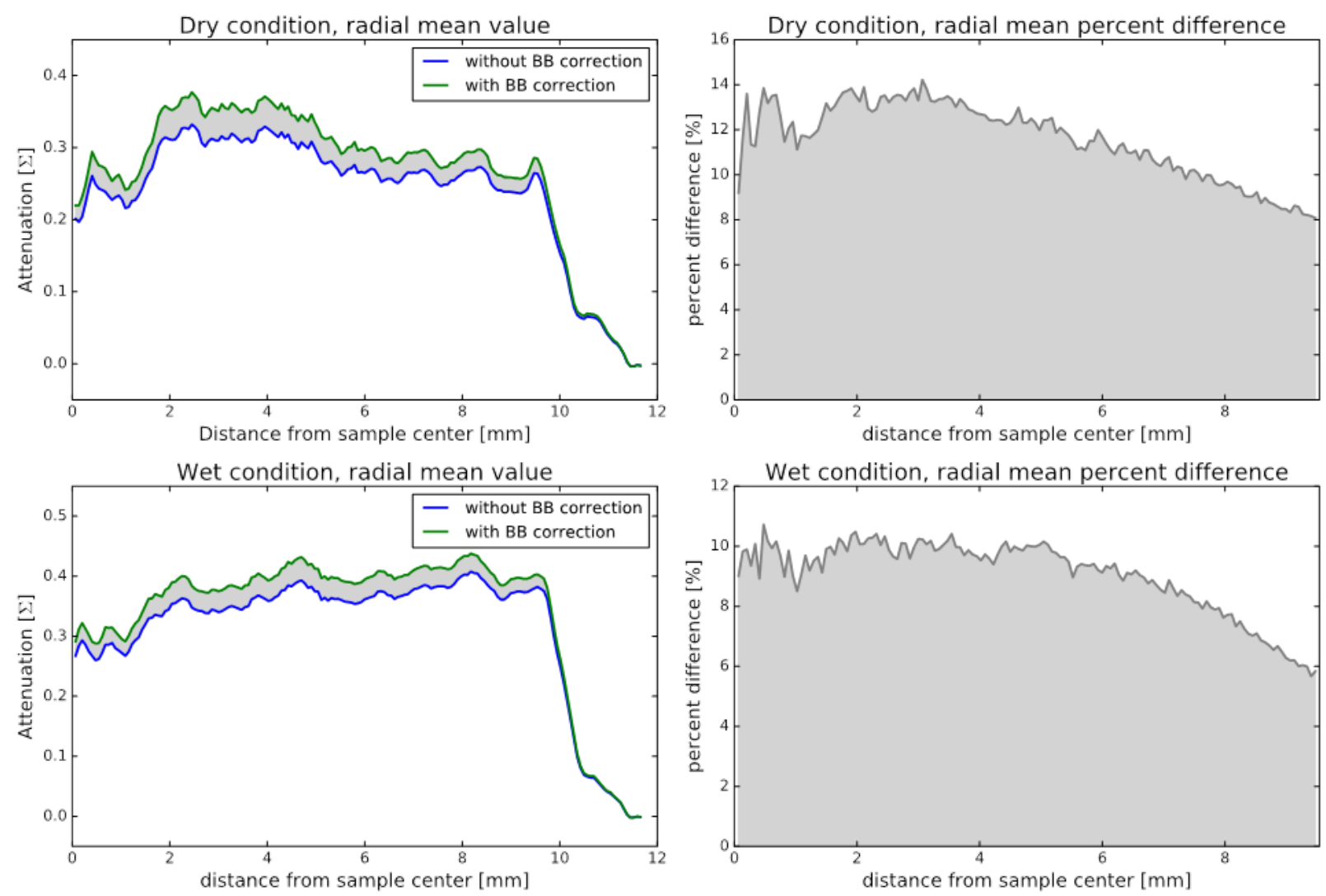

Figure 4: Radial mean values of attenuation coefficient, for dry and wet sample status. 


\section{Conclusions}

We have presented the beneficial effect of the BB approach for scattering and systematic bias correction in two experimental datasets. In both cases, the additive background that results in underestimated attenuation coefficient appears to be mitigated through the BB correction. In the time resolved radiographic study of wood samples, uncorrected images resulted in clear underestimation of water mass, while the BB corrected measurements resulted in good agreement with an independent reference measurement. For the on-the-fly tomographies, the BB correction proved effective in successfully compensating cupping, resulting from scattering bias, even though the effect was weak and not easy to identify without the BB approach. The effect was found to contribute about $5 \%$ in mean attenuation coefficient error and with higher significance for the wet condition. These results demonstrate that the BB correction is well applicable to time resolved kinetic neutron imaging studies in $2 \mathrm{D}$ as well as in 3D. As hydrogenous materials are strong incoherent neutron scatterers, this type of correction is indispensable for sensitive quantitative studies in neutron imaging, for example when the aim is to quantify the amount of water.

\section{Funding statements}

This project receives funding from the European Union's Horizon 2020 research and innovation programme under grant agreement No 654000.

The experiment on engineered soil sample was supported by Czech Science Foundation under grant No 17-21011S.

\section{References}

[1] P. Boillat, C. Carminati, F. Schmid, C. Grünzweig, J. Hovind, A. Kaestner, et al. Chasing quantitative biases in neutron imaging with scintillator-camera detectors: a practical method with black body grids. Optics Express. 26 (2018) 15769. https://doi.org/10.1364/OE.26.015769

[2] C. Carminati, P.Boillat, F.Schmid, P. Vontobel, J. Hovind, M. Morgano, M. Raventos, M. Siegwart, et al. Implementation and assessment of the black body bias correction in quantitative neutron imaging. PLoS ONE. 14(1), e0210300. https://doi.org/10.1371/journal.pone.0210300

[3] MuhRec release 4.0.1. doi:10.5281/zenodo.1438402

[4] D. Mannes, F. Schmid, T. Wehmann, E. Lehmann. Design and Applications of a Climatic Chamber for in-situ Neutron Imaging Experiments. Physics Procedia, 88 (2017), 200-207. https://doi.org/10.1016/j.phpro.2017.06.028

[5] E.H. Lehmann, P. Vontobel, L. Wiezel. Properties of the radiography facility NEUTRA at SINQ and its potential for use as European reference facility. Nondestructive Testing and Evaluation. 16 (2001) 191-202. https://doi.org/10.1080/10589750108953075

[6] Kaestner AP, Hartmann S, Kühne G, Frei G, Grünzweig C, Josic L, et al. The ICON beamline - A facility for cold neutron imaging at SINQ. Nuclear Instruments and Methods in Physics Research Section A: Accelerators, Spectrometers, Detectors and Associated Equipment. 659 (2011) p. 387-393. https://doi.org/10.1016/j.nima.2011.08.022

[7] M. Zarebanadkouki, A. Carminati, A. Kaestner, D. Mannes, M. Morgano, S. Peetermans, E.H. Lehmann, P. Trtik. On-the-fly neutron tomography of water transport into lupine roots Physics Procedia, 69 (2015), 292-298. https://doi.org/10.1016/j.phpro.2015.07.041 